\title{
From remote-controlled to self-controlled citizens
}

D. Helbing ${ }^{1,2, a}$

1 ETH Zurich, 8092 Zürich, Switzerland

2 Delft University of Technology, 2628 CD Delft, the Netherlands

Received 24 November 2016 / Received in final form 24 November 2016 Published online 18 January 2017

\begin{abstract}
The digital revolution will make data abundant and cheap. Moving from a time of darkness into a digital age with information overload, we will need suitable filters. However, those who build these filters will determine what we see. This creates possibilities to influence people's decisions such that they become remotely controlled rather than make their decisions on their own. Since omnibenevolent rule cannot be supposed and top-down control is flawed for several reasons, another approach is needed. It can be found with distributed control, collective intelligence and participation. "Nervousnet" will be presented as a feasible specimen of a Citizen Web.
\end{abstract}

\section{Remote-controlled citizens}

Moore's law - the exponential explosion of processing power and data production is transforming our economy and society. While processing power doubles every 18 months, data volumes double every 12 months. In one year we produce as much data as in all previous years, i.e. in the entire history of humankind.

But the digital revolution doesn't stop there. Fridges, coffee machines, tooth brushes, smartphones and smart devices are now equipped with communicating sensors. In ten years, 150 billion objects will connect with each other - and with 10 billion people. This emerging 'Internet of Everything' will generate data volumes that double every 12 hours rather than every 12 months. How will this impact our society?

Data will be abundant and cheap. Most money will be made with computer algorithms that distill useful information from raw data, and actionable knowledge from information. Imagine a situation where the amount of data about our world determines how well we can see and understand it. It, then, becomes clear that we are moving from a time of darkness, where we did not see enough to make good decisions, into a digital age where we tend to be blinded by information, i.e. suffering from extreme information overload. To master this situation, we will need suitable filters, something like 'digital sun glasses'. Whoever builds these filters will determine what we see [1]. This creates possibilities to influence people's decisions in such subtle a way that they would consider these decisions their own, while they have been actually remotely controlled.

\footnotetext{
${ }^{a}$ e-mail: dirk.helbing@gess.ethz.ch
} 
Until today, science hardly understands the complexity of human decision-making. Our habits, routines and social interactions, however, make us surprisingly predictable [2], which is monetized by companies such as Recorded Future and Palantir. By now, about 3000 to 5000 metadata have been collected about every one of us. ${ }^{1}$ This makes it easy to manipulate our decisions and feelings $[3]^{2}$. In fact, our behavior is increasingly steered by technology. Think of recommender systems, individually customized ads, and personalized search results.

When the thickness is not equal to one of these discrete values, the film locally adopts one of the "allowed" values everywhere. Due to overall volume conservation, the upper layer is incomplete and has been described in terms of "islands" or "holes".

\section{Ruling like a 'wise king'?}

Could one use this technology to align individual interests (the 'user optimum') with the 'system optimum' and run the world in an optimal way? It has become popular to think that personal data may be used to 'nudge' people's decisions so that they will behave in a healthier and environmentally friendly way. This comes under the banner of 'soft' or 'libertarian paternalism' [4]. However, the same technology may also be used to promote nationalism, hate against minorities, or more favorable election outcomes. $^{3}$

Therefore, it is crucial, who is entitled to use these technologies and how. As long as ethical scrutiny, sufficient transparency and democratic control are lacking, 'nudging' might well create totalitarian powers. If knowledge is power, this even seems to imply 'God-like' attributes: could 'omnipotence' be reached by knowledge of everything (i.e. by massive centralized collection of personal data in ways that ignore privacy and rights of informational self-determination)? Is this what governments and Big Data companies are striving for, fueled by thousands of metadata summarizing our personality?

In fact, some countries have started to experiment with a data-driven, 'cybernetic society', i.e. they apply concepts from control theory to run their societies. The first attempt in Chile in the early 70s ended in tragedy. ${ }^{4}$ Since then, information technology has been much advanced. Singapore, which considers itself a 'social laboratory' [5], is running its country in a top-down way, pretty much like a company. ${ }^{5}$ The efficiency of this decision-making approach is believed to have boosted the development of the country. Singapore is now seen as a successful example of a data-driven society and often as a model for the rest of the world.

\footnotetext{
1 See https://www. democraticmedia.org/acxiom-every-consumer-we-have-more-5000attributes-customer-data.

2 This experiment was highly controversial, see http://www.wsj.com/articles/ furor-erupts-overfacebook-experiment-on-users-1404085840.

3 Surveillance-based manipulation: How Facebook or Google could tilt elections, see http://arstechnica.com/security/2015/02/surveillance-based-manipulation-howfacebook-or-google-could-tilt-elections/; some experts believe that up to 20 percent of votes may be changed by nudging.

4 A military coup supported by a foreign power stopped the development, and president Salvador Allende committed suicide, see E. Medina, Cybernetic Revolutionaries (MIT Press, 2011) and also http://www.spiegel.de/einestages/projekt-cybersyn-stafford-beersinternet-vorlaeufer-in-chile-a-1035559.html.

5 This raises the question, whether the 'Singaporean model' will be sustainable, given that 40 percent of the top 500 companies disappear within 10 years. In fact, the public support for the governing political party has steadily declined over the last elections.
} 
Among decision-makers around the world, the 'China model' has also become very popular. It impresses by the quick realization of large-scale infrastructural projects. This is possible, as the concerns of citizens are paid less attention to. However, abandoned 'ghost malls' have been built too, and some cities have become almost unlivable due to high levels of air pollution. Therefore, China is now also engaged in a data-driven approach. For example, the Internet service company Baidu, the Chinese equivalent of Google, invited the military to join the 'China brain project', which uses 'deep learning' algorithms to predict peoples' behaviors based on their Internet searches. ${ }^{6}$ China's government has also initiated a project that rates the behavior of its citizens. ${ }^{7}$ Loans and jobs will soon depend on personal scores, web links clicked - and political opinions. All of this might happen with the very best intentions: Big Data now seems to empower governments to overcome irrationality and partial interests, and to rule like a 'benevolent dictator' or 'wise king'. But would this really work?

\section{Why the 'wise king' approach will fail}

My assessment is that, in complex societies, a 'wise king' approach might rather cause chaos than order. The Information Box summarizes the principal limitations of data-driven top-down control. A major concern is also the vulnerability to hacking, corruption, and error, which is particularly pronounced for centralized information systems and approaches. Sooner or later, organized criminals, terrorists or extremists would manage to get them under control.

An even bigger issue is the fact that a 'wise king' would have to decide for a goal function to maximize, but there is no scientific method to figure out what is the right one. Should it be GDP per capita, power, sustainability, average life span, peace, quality of life, or something else? We must understand that 'omnibenevolence' does not exist. Therefore, Immanuel Kant, the father of the age of enlightenment concluded that a government that decides about its citizens' pursuit of happiness is a despot. ${ }^{8}$ This is also the basis of the U.S. Declaration of Independence. ${ }^{9}$

There are three major flaws of top-down control: First, its micromanagement overwrites cultural cues, which have guided peoples' decisions in the past. This interferes with the self-organization on lower levels of society (families, companies, non-profit organizations, etc.), and it may destroy the basis of social stability and order. Second, influencing peoples' decisions locally narrows down the variance of choices, which undermines the 'wisdom of the crowd effect', leading to bad decision outcomes [6,7]. Third, reducing socio-diversity and the pluralistic choice of goal functions is similarly bad as losing bio-diversity. The economy and society

\footnotetext{
${ }^{6}$ Baidu welcomes China's military to join China Brain project on AI systems, see http:// www . wantchinatimes. com/news-subclass-cnt . aspx?id=20150307000015\&cid=1101.

7 China rates its own citizens - including online behaviour, see http://www. volkskrant. $\mathrm{nl} /$ buitenland/china-rates-its-own-citizens-including-online-behaviour a3979668/; China: Kontrolle über alles, see http://www.zeit.de/politik/ausland/ 2015-07/china-plangesellschaft-xi-jinping.

8 See http://oll.libertyfund.org/titles/kant-kants-principles-of-politicsincluding-his-essay-on-perpetual-peace/simple and http://plato.stanford.edu/ entries/kant-social-political/.

9 It states: "We hold these truths to be self-evident, that all men are created equal, that they are endowed by their Creator with certain unalienable Rights, that among these are Life, Liberty and the pursuit of Happiness" (after president Thomas Jefferson).
} 
should be understood like ecosystems with millions of interdependencies [8]. Thus, if diversity is reduced, the economic development and success of a society are affected [9]. This can lead to systemic malfunction, political instability, collapse, and war [10-12]. Moreover, personalized information creates a 'filter bubble' effect [1], such that everyone is less exposed to other opinions. As a consequence, different groups of people have difficulties to understand and tolerate each other. This has led to increasing discrimination and polarization [13], causing extremism and societal conflict.

In fact, political satisfaction has decreased in many areas of the world, and there is no sign that we have become better in mastering global threats since Big Data is being used for control. We are confronted with an economic and public spending crisis, financial and political instability, increasing dangers of large-scale international conflict or cyber wars, and climate change with a mass extinction of species, to mention just a few of the unsolved problems. Data-driven top-down control may certainly have short-term benefits, but it creates unwanted side effects, which are very costly and may eventually end in disaster. In particular, while the digital revolution has made our lives more comfortable and interesting, it has so far failed to produce the large-scale macroeconomic benefits, which would justify the 'creative destruction' of our current socio-economic system that we currently see. As Apple co-founder Steve Wozniak pointed out: "Computers are going to take over from humans, no question ... the future is scary and very bad for people ... If we build these devices to take care of everything for us, eventually they'll think faster than us and they'll get rid of the slow humans to run companies more efficiently ..." If people shall still be able to spend significant amounts of money on services and goods, we will have to create about 50 percent new jobs within 10 to 20 years [14]. This makes it clear that we will have to totally re-invent our economy and society, and we have to do it quickly.

\section{A better approach: Distributed control, collective intelligence, participation}

As Albert Einstein (1879-1955) said: "We cannot solve our problems with the same kind of thinking that created them." To benefit from the digital revolution, we need an entirely new approach. It is clear that those countries will be leading, which manage to use their full capacity, i.e. mobilize the ideas, skills and resources of its citizens best. So, how can we create a win-win-win situation of business, politics and citizens? We need to build an ecosystem of socio-economic activities, where each new idea, product and service creates opportunities for further ideas, products and services. An open data strategy ${ }^{10}$. a "new deal on data" ${ }^{11}$, open innovation, interoperability and suitable intellectual property rights can catalyze such an ecosystem and boost economic growth. This also requires 'networked thinking' and a participatory approach. Given that, in future, a decreasing fraction of data can be further processed, many decisions will have to be taken locally. This speaks for a 'distributed control' approach,

\footnotetext{
10 Open Data in the G8: A Review of Progress on the G8 Open Data Charter, see http:// www2.datainnovation.org/2015-open-data-g8.pdf. A report by McKinsey \& Co. estimates the additional value of Open Data to be of the order of $\$ 3-5$ trillion per year: http://www.mckinsey.com/insights/business_technology/open_data_unlockinginnovation_and_performance_with_liquid_information.

11 World Economic Forum, Personal Data: The Emergence of a New Asset Class, see http://www . weforum.org/reports/personal-data-emergence-new-asset-class .
} 
which is perfectly compatible with autonomous decision-making, democratic values and free entrepreneurship.

Given the many interdependencies and feedback effects, it's impossible for anybody and even supercomputers to grasp the full complexity of our world. To cope with this complexity, we must learn to create 'collective intelligence'. First, this requires trustworthy information exchange and unbiased, reliable, high-quality data for everyone, i.e. just the opposite of manipulation. Second, it requires us to take the knowledge and ideas of more people on board [7], for example through citizen science, suitable online deliberation platforms, and other crowd sourcing approaches. We also need a pluralistic modeling approach [15], which considers and combines multiple perspectives. Therefore, everyone should be able to use their own information filters (e.g. run the search algorithms of their choice). In fact, diversity is key for high innovation rates, collective intelligence, and societal resilience to disruptions [16]. Therefore, pluralism shouldn't be seen as a concession to citizens. It is as a functional precondition for complex societies to thrive.

Pluralism hedges the risks to humanity, as it maximizes the chance that, whatever happens, suitable solutions would somewhere exist. Therefore, we should also engage in what I call 'the cultural genome project'. This would aim to reveal and formalize the unwritten rules and hidden success principles of the cultures of the world (e.g. how to build 'social capital' such as trust, which largely benefits the economy, too). This would allow us to use and recombine these rules in new ways, and to turn cultural diversity into an asset, helping us to create new value and new jobs.

Today, we have quite some difficulties to deal with diversity, as our efforts to standardize products and services as well as our debates about migration and minorities show. However, 'digital assistants' will soon be able to support us. Apps for real-time translation already help us to overcome cultural barriers. In future, they will guide us to engage in favorable interactions, avoid unfavorable ones, and create win-win situations, which we would otherwise have overlooked [16]. But where would we get the data for this?

\section{Nervousnet: A decentralized digital nervous system}

In the same way as we have built public roads to promote the industrial age and public schools to fuel the service society, we need public information systems and digital literacy to support everyone's decision-making and the digital era to come. Remember that the open standards of the World Wide Web (WWW) have unleashed a digital economy worth many billion dollars, and that participatory projects such as Linux, Wikipedia and OpenStreetMap have created opportunities for everyone. I propose, therefore, to build a Planetary Nervous System that uses the sensor networks behind the Internet of Everything, including those in our smartphones, to measure the world around us and build a data commons together. The question is how to do this while respecting privacy and minimizing misuse. It is time to learn this now.

The Nervousnet project, a spin-off of the FuturICT project, has started to work on such an open and participatory, distributed information platform for real-time data. $^{12}$ Nervousnet is an open source project, which believes in the importance of privacy, informational self-determination and trust. If you download the Nervousnet app to your smartphone, you can choose to turn about 10 different sensors separately on or off, such as the accelerometer, light or noise sensors. You can measure data

12 See http://www.nervous.ethz.ch/ and http://www.futurict.eu, also https://www. youtube.com/watch?v=BKcWPdSUJVA. The Nervousnet app can be downloaded via Apple's app store and Google's play store. You can contact us at nervousnet@ethz.ch. 
about your environment for yourself (kept on your smartphone) or share it with others (as decided by yourself). External sensors for 'smart home' and other applications can be added (for example, weather or health sensors). To maximize informational self-control, the user can also determine the recording rate, and soon also the storage time after which the data will be deleted. Shared data are anonymized. In addition, we are working on data encryption and plan to add a personal data store [17], which will allow you to determine what kind of data you want to share with whom, for what purpose, and for what period of time.

Nervousnet will be run as a Citizen Web, built and managed by its users. It will allow all developers to add measurement procedures and apps. For example, you may run games, scientific measurement projects, or business applications on top of the Nervousnet platform. So, anyone can add data-driven services and products. For security and conceptual reasons (such as scalability and fault-tolerance), Nervousnet is based on distributed data and control. To promote responsible use, Nervousnet will integrate reputation, qualification, and community-based self-governing mechanisms, determining accessible sensors, data volume and functionality. All this is intended to catalyze a novel information, innovation and production ecosystem to create societal benefits, business opportunities, and new jobs.

Nervousnet will offer five main functionalities. First, it will empower us to collectively measure the world around us in real time. For example, it will allow us to quantify external effects of our interactions with the environment and others (such as noise and emissions, but also economic, social and immaterial value created). Such measurements can help to create a circular economy and more sustainable systems.

Second, Nervousnet will help to create awareness about the problems and opportunities around us. It will warn us of the side effects of certain decisions and actions (e.g. the amount of $\mathrm{CO}_{2}$ emissions produced) and support us in identifying and implementing better alternatives (e.g. how to use public transport comfortably).

Third, the Nervousnet data stream will establish something like a 'CERN for society'. It will allow us to reveal the hidden regularities and forces underlying socio-economic change. This will create the knowledge to establish a Global System Science [18], which is needed to master our future in a highly complex and interdependent world.

Fourth, the combination of this knowledge with real-time data will enable us to build self-organizing systems, using real-time feedbacks. With the right kinds of interactions, a complex dynamical system can create a huge variety of self-stabilizing structures, properties and functions in a way that is self-organized and enormously efficient. For example, self-controlled traffic lights, which flexibly respond to local vehicle flows, can dramatically reduce urban congestion compared to the classical, centralized control approach [19,20]. By using the Internet of Everything many production processes can similarly benefit (often summarized under the label 'industry 4.0').

Fifth, Nervousnet will support a network of distributed artificial and human intelligence. Digital assistants will support us not only in everyday situations, but also in bringing knowledge, ideas, and resources efficiently together. By creating such 'collective intelligence', we will be able to master the combinatorial complexity of our increasingly interdependent world much better (see above).

Finally, to fully unleash the socio-economic benefits, we will need a micro-payment system, too. However, given that money is mainly a coordination mechanism to decide about the distribution of scarce material resources, we can build a better system today. One the one hand, to enable self-organization and to be consistent with pluralism, a multi-dimensional incentive and exchange system is needed [21]. On the other hand, money can not only be made by cutting costs (i.e. rationalization), but also by the co-creation of information-based and cultural goods. While the limitation of 


\section{Government 1.0}

\section{Government 2.0}

Government 3.0

Government 4.0
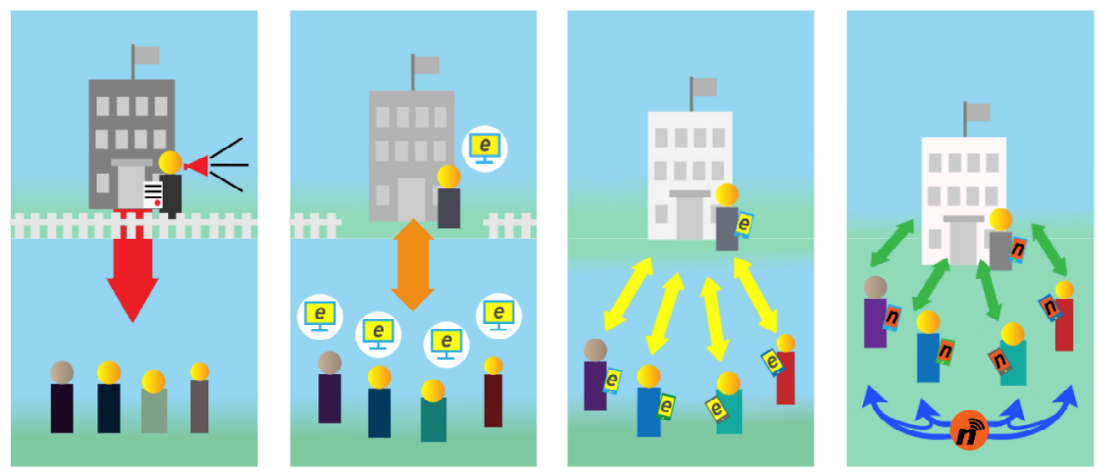

Fig. 1. Illustration of the evolution of government models. Government 1.0 tells people what to do and is separated from its citizens. Government 2.0 uses electronic means to engage in a two-way interaction with its citizens. Government 3.0 engages in individually fitting interactions, services, and solutions. This is currently being implemented in South Korea. Government 4.0 also builds on the coordination among its citizens, and on co-creation.

material resources implies a subtractive kind of money (if I want to have more, someone else will have to own less), the information-based economy allows for additive money (if I want to have more, you can also have more - this is the natural basis of shared knowledge and culture). Information can be multiplied as often as we like, and it can be used in an unlimited number of ways. Therefore, the digital economy doesn't have to be a zero sum game. If we create the right framework for the digital society and economy to come ${ }^{13}$, this can reduce conflict and foster an age of prosperity for everyone. Why don't you help to create this framework for a better future?

\section{Instead of a conclusion: Limitations of data-driven top-down control}

In practice, large-scale system optimization and successful top-down control are limited by at least four factors.

First, data volume grows faster than processing power, implying that a growing share of data will never be processed. This creates a "flashlight effect": we may see anything we want, but need to know what to pay attention to.

Second, due to limited communication bandwidth, an even smaller fraction of data can be processed centrally, while in complex dynamical systems every little detail can matter [22]. Therefore, centralized optimization attempts ignore a lot of local information, which is needed to produce good solutions.

Third, algorithmic complexity can prevent real-time optimization, such that decentralized control approaches may perform better. This has been shown for city traffic: self-organised traffic lights controlled by local traffic flows can perform better than those run by a traffic control center [19,20].

Fourth, further problems may be caused by poor models or 'over-fitting', parameter sensitivity, spurious correlations, meaningless patterns, noise and classification errors - all very common problems in Big Data analytics [23].

Figure 1 shows different government modes and how technology can be used to create other modes. Model Government 4.0 is the one that is implied by Nervousnet.

\footnotetext{
13 Note that we can also reduce scarcities of material resources by coordinated temporary use, as it is now enabled by the new approaches of the 'sharing economy'.
} 


\section{References}

1. E. Pariser, The Filter Bubble: What the Internet Is Hiding from You (Viking/Penguin, 2011)

2. C. Song, Z. Qu, N. Blumm, A.-L. Barabasi, Science 327, 1018 (2010)

3. A.D.I. Kramer, J.E. Guillory, J.T. Hancock, Experimental evidence of massive-scale emotional contagion through social networks, Proc. Natl. Acad. Sci. USA 111, 8788 (2014)

4. R.H. Thaler, C.R. Sunstein, Nudge: Improving Decisions About Health, Wealth, and Happiness (Penguin, New York, 2008)

5. S. Harris, Foreign Policy, http://foreignpolicy.com/2014/07/29/the-sociallaboratory/ (2014)

6. J. Lorenz, H. Rauhut, F. Schweitzer, D. Helbing, PNAS 108, 9020 (2011)

7. S.E. Page, The Difference: How the Power of Diversity Creates Better Groups, Firms, Schools, and Societies (Princeton University, 2008)

8. R.M. May, S.A. Levin, G. Sugihara, Nature 451, 893 (2008)

9. C.A. Hidalgo, B. Klinger, A.-L. Barabasi, R. Hausmann, Science 317, 482 (2007)

10. J. Diamond, Collapse: How Societies Choose to Fail or Succeed (Penguin 2011)

11. J.A. Tainter, The Collapse of Complex Societies (Cambridge University, 1990)

12. D. Helbing, Jusletter IT, 21 May 2015

13. C. Andris et al., Plos one 10, e0123507 (2015)

14. C.B. Frey, M.A. Osborne, The future of employment: How susceptible are jobs to computerisation? http://www.oxfordmartin.ox.ac.uk/downloads/academic/futureof-employment.pdf (2013)

15. D. Helbing, Science and Culture 76, 315 (2010)

16. D. Helbing, The automation of society is next: How to survive the digital revolution, http://dx.doi.org/10.2139/ssrn. 2694312 (2015)

17. Y.-A. de Montjoye, E. Shmueli, S.S. Wang, A.S. Pentland, Plos one 9, e98790 (2014)

18. D. Helbing, Nature 497, 51 (2013)

19. S. Lämmer, D. Helbing, JSTAT 2008, P04019 (2008)

20. D. Helbing, Evol. Inst. Econ. Rev. 10, 3 (2013)

21. D. Helbing, Qualified money: A better financial system for the future, http://futurict . blogspot.ch/2014/10/qualified-money-better-financial-system.html (2014)

22. I. Kondor et al., J. Econ. Interact. Coord. 9, 203 (2014)

23. D. Helbing, Thinking Ahead: Essays on Big Data, Digital Revolution, and Participatory Market Society (Springer, 2015)

Open Access This is an Open Access article distributed under the terms of the Creative Commons Attribution License (http://creativecommons.org/licenses/by/4.0), which permits unrestricted use, distribution, and reproduction in any medium, provided the original work is properly cited. 